\title{
Species Diversity, Antifungal Susceptibility, and Virulence Attributes of Candida Colonising the Oral Cavities of Adult Diabetic Patients
}

\author{
Deepa Anil Kumar, ${ }^{1}$ Sumathi Muralidhar, ${ }^{2}$ Krishna Biswas, ${ }^{3}$ \\ Uma Banerjee, ${ }^{4}$ Seemi Farhat Basir, ${ }^{1}$ and Luqman Ahmad Khan' \\ ${ }^{1}$ Department of Biosciences, Jamia Millia Islamia, New Delhi 110 025, India \\ ${ }^{2}$ Apex Regional S T D Centre, VMMC and Safdarjung Hospital, New Delhi 110 029, India \\ ${ }^{3}$ Department of Endocrinology, VMMC and Safdarjung Hospital, New Delhi 110 029, India \\ ${ }^{4}$ All India Institute of Medical Sciences, New Delhi 110 029, India \\ Correspondence should be addressed to Luqman Ahmad Khan; 1khan@jmi.ac.in
}

Received 26 June 2014; Revised 29 August 2014; Accepted 2 September 2014; Published 18 September 2014

Academic Editor: Ángel Domínguez

Copyright (c) 2014 Deepa Anil Kumar et al. This is an open access article distributed under the Creative Commons Attribution License, which permits unrestricted use, distribution, and reproduction in any medium, provided the original work is properly cited.

\begin{abstract}
Oral candidiasis is a common occurrence in diabetic patients. Species of Candida isolated from these infections and their virulence pattern undergo changes over time and require periodic assessments. Objective of this study was to determine changes in the spectrum of Candida species colonizing oral cavity, their antifungal susceptibility patterns, and virulence attributes, in adult diabetic patients. Oral swabs were collected from 100 patients with diabetes mellitus (DM) and an equal number of healthy controls. Specimens were cultured for Candida and species were identified, according to standard protocols. Of 100 diabetic patients, 42 were colonized by yeasts with $C$. albicans as the predominant species (51\%). Non-Candida albicans Candida (NCAC) species accounted for $47 \%$ of the specimens, with C. tropicalis being the commonest. Among healthy controls, 23 were colonized by Candida species, of which C. albicans was predominant. Results obtained indicate that C. albicans continues to be the predominant species in oral cavities of diabetic patients. Candidal carriage was significantly associated with duration of diabetes and fasting blood sugar levels. Virulence attributes, proteinase and phospholipase secretion, and biofilm formation were significantly higher in DM group.
\end{abstract}

\section{Introduction}

Diabetes mellitus (DM) is a growing public health concern and a common chronic metabolic disease worldwide, and India is identified as the diabetes capital of the world [1]. World Health Organization considers diabetes as one of the major noncommunicable disease [2]. Among the 10 countries estimated to have the largest number of cases with diabetes, India is leading the list at present [3]. Of the two types of diabetes, type 1 (also known as insulin dependent) and type 2 (also known as insulin independent), type 2 is more prevalent. DM is associated with various inflammatory diseases and soft tissue pathologies in the oral cavity; however, awareness of these complications is lacking worldwide [4]. The frequent occurrence of Candida infections has been recognized for many years and oral candidiasis in particular is thought to be more prevalent among these individuals [57].

Studies on the relationship between DM and Candida are often contradictory [8]. There is a paucity of data on carriage rate of Candida species in DM patients, along with its virulent attributes and antifungal resistance patterns. Hence, the present study was designed to evaluate spectrum of yeast species in oral cavity of Type 2 DM patients on various antidiabetic treatment modalities, in relation to duration of diabetes. The study was also conceived to investigate the 
antifungal resistance patterns, hydrolytic enzyme production, and biofilm formation, on the isolates to get a baseline data.

\section{Subjects and Methods}

One hundred diabetic patients, aged between 30 and 73 years, registered in the diabetic clinic of Safdarjung Hospital, New Delhi, were included in the study. Oral swabs were collected from consecutive type 2 diabetic patients attending the clinic on the particular day of sample collection. Specimens were collected once in a week, for a duration of six months (September 2011 to March 2012). The control group included 100 healthy volunteers who were age and sex matched, without history of diabetes. Subjects excluded were those with pregnancy, on oral contraceptives, on antibiotics, and with history of treatment with antifungals during previous six months. All subjects included in the study were informed about the procedures and possible outcomes and their samples were collected after an informed written consent. The study protocol was approved by the Institutional Ethics Committee of Safdarjung Hospital (no. 26-11-EC (21/31)).

Clinical and demographic data collected at the time of sample collection from each patient included age, gender, blood glucose profile, duration of diabetes, and treatment status. The subjects were divided into three groups based on their antidiabetic treatment (Group-1: those controlled solely on oral antidiabetics such as sulfonyureas or metformin; Group-2: those controlled on insulin regimen; Group 3: those controlled on oral antidiabetics + insulin regimen).

Two swabs were collected from each subject by depressing the tongue and gently rubbing the surface of gum, tooth, tonsils, and tongue using sterile cotton swabs. Swabs were transported to the lab immediately and processed further without any delay. First swab was inoculated on Sabouraud's Dextrose Agar (SDA) and was incubated at $37^{\circ} \mathrm{C}$ for up to $7 \mathrm{~d}$ and was observed daily for growth, while the second swab was used to prepare a smear for Gram staining. In case of positive growth, yeast identification was done by conventional and standard methods [9]. Nonidentical colonies obtained from a single patient were subcultured separately. To differentiate Candida albicans from C. dubliniensis, all C. albicans isolates (confirmed by conventional methods) were subjected to growth at $45^{\circ} \mathrm{C}$ and Tween 80 hydrolysis test along with the ATCC control strains [10]. When conventional method failed to identify unusual isolates, they were verified by the use of automated Vitek 2 YST system (bioMerieux, France).

Subsequent to species identification, antifungal susceptibility testing was performed on all the isolates using antifungal agents including Fluconazole (FL), Ketoconazole (KE), Voriconazole (VO), and Amphotericin B (AP) by E-Test strip method. This was performed by inoculating a portion of the isolated colony in normal saline, adjusting its turbidity to 0.5 McFarland standard. The suspension was then spread over the surface of a predried RPMI+ $2 \%$ glucose agar media, using a sterile cotton swab. E test strips were applied using a sterile forceps and MICs were determined after 24 and $48 \mathrm{~h}$ of incubation at $37^{\circ} \mathrm{C}$ [11]. The MIC breakpoints recommended by Clinical Laboratory Standards Institute (CLSI) M27-A3 were followed (Table 4) [12].

\section{Assay for Extracellular Proteinase and Phospholipase}

3.1. Preparation of the Inoculum. A single colony was inoculated in $10 \mathrm{~mL}$ of yeast extract peptone dextrose broth and incubated at $37^{\circ} \mathrm{C}$ for $18 \mathrm{~h}$; the inoculum was then transferred to a centrifuge tube and centrifuged at $3000 \mathrm{rpm}$ for $5 \mathrm{~min}$. The supernatant was discarded and the pellet obtained was washed with sterile distilled water. The pellet was resuspended in sterile normal saline and centrifuged to remove any residual media. Suspension containing $1 \times 10^{6}$ yeast cells $\left(1.25 \mathrm{OD}_{550} / \mathrm{mL}\right)$ was prepared in sterile normal saline and processed further.

Proteinase production by the isolates was determined on a medium containing bovine serum albumin (BSA) according to standard method. Nine hundred millilitres of media was prepared which contained yeast nitrogen base without amino acid: $2 \mathrm{~g}$; ammonium sulphate: $1.45 \mathrm{~g}$; glucose: $20 \mathrm{~g}$; agar: $20 \mathrm{~g}$. The media was autoclaved at $121^{\circ} \mathrm{C}$ for $20 \mathrm{~min}$. Two grams BSA was dissolved in $100 \mathrm{~mL}$ of distilled water and sterilized by filtration. Sterilized BSA was slowly added to the autoclaved and cooled media and poured into petri dish. On this media $1 \mu \mathrm{L}$ of the inoculum was deposited at equidistant points and allowed to dry at room temperature. The plates were incubated at $37^{\circ} \mathrm{C}$ for $3-4 \mathrm{~d}[13,14]$. Each isolate was inoculated in triplicate. C. albicans ATCC 10231 and ATCC 10261 were used as positive controls. Candida parapsilosis ATCC 22019 was used as a negative control. Proteinase activity was determined by calculating the zone of digestion $\left(P_{z}\right)$ using the formula-ratio of the diameter of the colony to the sum of diameter of the colony and the zone (in $\mathrm{mm})[13,14]$.

Candida isolates were screened for production of extracellular phospholipase activity by growing them on egg yolk agar and measuring the size of the zone of digestion $[13,14]$. Briefly, the egg yolk medium consisting of agar: 20 g; peptone: 10 g; glucose: $30 \mathrm{~g}$; $\mathrm{NaCl}: 57.3$ g; $\mathrm{Cacl}_{2}: 0.55 \mathrm{~g}$; distilled water added to $900 \mathrm{~mL}$, was autoclaved at $121^{\circ} \mathrm{C}$ for $20 \mathrm{~min}$. One hundred millilitres of egg yolk was slowly added to the autoclaved and cooled media and poured into sterile petridish. On this media $2 \mu \mathrm{L}$ of the inoculum was deposited at equidistant points and allowed to dry at room temperature. The plates were incubated at $37^{\circ} \mathrm{C}$ for $3-4 \mathrm{~d}$ $[13,14]$. Candida albicans ATCC 10231 and ATCC 10261 were used as positive controls. Candida glabrata ATCC 90030 was used as a negative control. $P_{z}$ was measured by dividing the diameter of the colony by the sum of diameter of the colony and the zone $[13,14]$, as shown in Figure 4.

Biofilm formation was determined spectrophotometrically by one of the methods described by Tumbarello et al. [15], with minor modifications. Briefly, Sabouraud dextrose broth $(\mathrm{SDB})$ was prepared with a final concentration of $8 \%$ glucose. Candida isolates were grown on SDA for $24 \mathrm{~h}$ and saline washed suspensions of each isolate were prepared. The turbidity of each suspension was adjusted to a concentration of $3 \times 10^{7} \mathrm{CFU} / \mathrm{mL}$ by spectrophotometer. To each well of the microtitre plate containing $180 \mu \mathrm{L}$ of SDB, $20 \mu \mathrm{L}$ of the suspension was added. After incubating at $37^{\circ} \mathrm{C}$ for $90 \mathrm{~min}$ (adhesion phase), medium containing planktonic cells was 
discarded and each well was gently washed with PBS. For biofilm formation, fresh medium was added and the plates were incubated at $37^{\circ} \mathrm{C}$ for $48 \mathrm{~h}$. After incubation, the wells were washed twice with PBS to remove any planktonic cells and $200 \mu \mathrm{L}$ of PBS was added. The biofilm was measured directly by the spectrophotometric reading at $405 \mathrm{~nm}$ with a microtitre plate reader. The percent transmission $(\% T)$ was calculated subtracting the $\% T$ value of each test sample from the $\% T$ of the reagent blank to obtain $\% T_{\text {bloc }}$. Biofilm formation was scored based on their $\% T$ values as either negative $\left(\% T_{\text {bloc }}<10\right)$ or graded as $1+\left(\% T_{\text {bloc }} 10-20\right)$, $2+$ $\left(\% T_{\text {bloc }} 20-35\right), 3+\left(\% T_{\text {bloc }} 35-50\right)$, and $4+\left(\% T_{\text {bloc }} \geq 50\right)$. The isolates were further classified into low biofilm producers $(1+)$ and high biofilm producers $(2+, 3+$, or $4+)$ [15].

3.2. Data Analysis. Data analysis was performed by Student's $t$-test, Chi square test, and Pearson correlation test, using GraphPad prism software. A $P$ value of $<0.05$ was considered significant.

\section{Results}

Out of 100 oral swabs obtained from the diabetic patients, 42 yielded different yeasts (42\%), of which 35 swabs yielded single species and seven yielded two or more species, giving a total of 51 isolates. Candida albicans (51\%) was the predominant species isolated. NCAC species accounted for $47 \%$. C. tropicalis (15\%) and C. krusei (14\%) showed predominance over the other NCAC isolates, whereas out of 100 samples from the healthy controls only 23 yielded the growth of Candida, with a predominance of C. albicans (18) followed by C. glabrata (3), C. parapsilosis (1), and C. tropicalis (1) (Figure 1).

Out of 50 subjects who were only on oral antidiabetic drugs (Group 1), 20 yielded different Candida species (40\%), of which 19 yielded single species and one yielded more than one species, giving a total of 21 isolates. In 29 diabetic patients who were on insulin regimen (Group 2), growth was observed in 12 cases (41.38\%), with eight yielding single isolates and four yielding multiple isolates, giving a total of 16 isolates. In the third group (Group 3), 10 (47.61\%) yielded different species of Candida, out of 21 subjects investigated for its carriage. Of these seven yielded only single isolates and three yielded multiple isolates, giving a total of 14 isolates (Figure 2), (Table 1).

Among patients with duration of diabetes one to 10 years, 27 yielded growth, of which 21 yielded single isolates and six yielded multiple isolates, giving a total of 34 . A total of 13 isolates were obtained from 11 patients having diabetes history of 11-20 years. Four out of seven patients with diabetes history of more than 20 years yielded growth (Figure 3). The present study revealed a positive correlation between oral yeast carriage in relation with fasting blood sugar level (Table 2). But no such correlation was observed in relation with age of diabetic patients (Table 3 ).

The in vitro antifungal susceptibility of the isolates revealed that none of the $C$. albicans isolated from both the control and the diabetic group showed resistance to any of the agents tested. NCAC isolated from control group also yielded

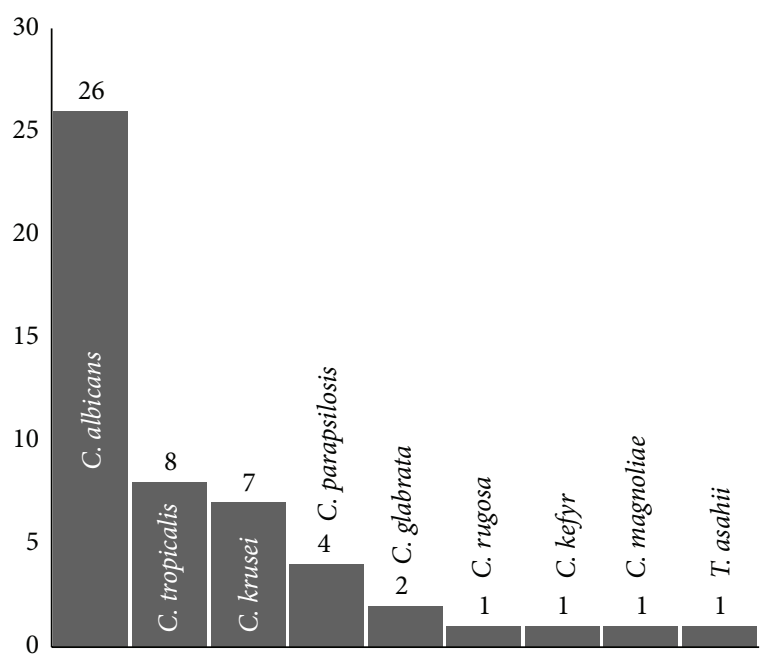

FIGURE 1: Species distribution of yeasts in the oral cavity of diabetic patients $(n=51)$.

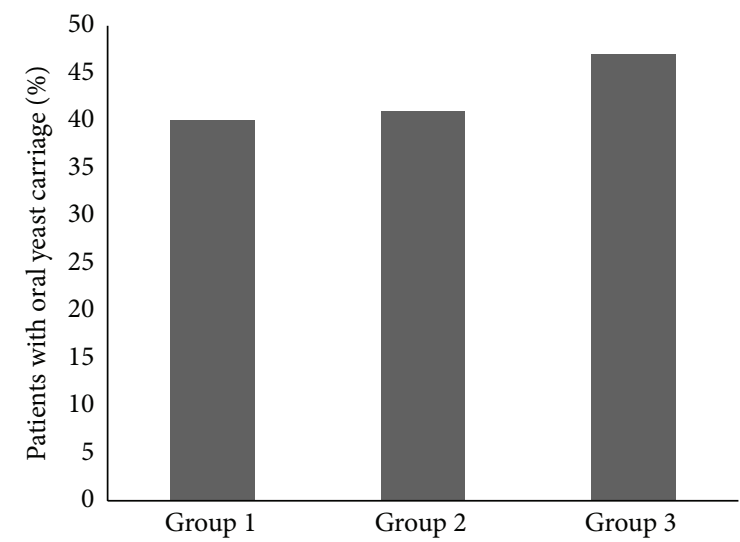

FIGURE 2: Oral yeast carriage in relation to antidiabetic treatment. Group-1: controlled solely on oral antidiabetics; Group-2: controlled on insulin regimen; Group 3: controlled on oral antidiabetics + insulin regimen.

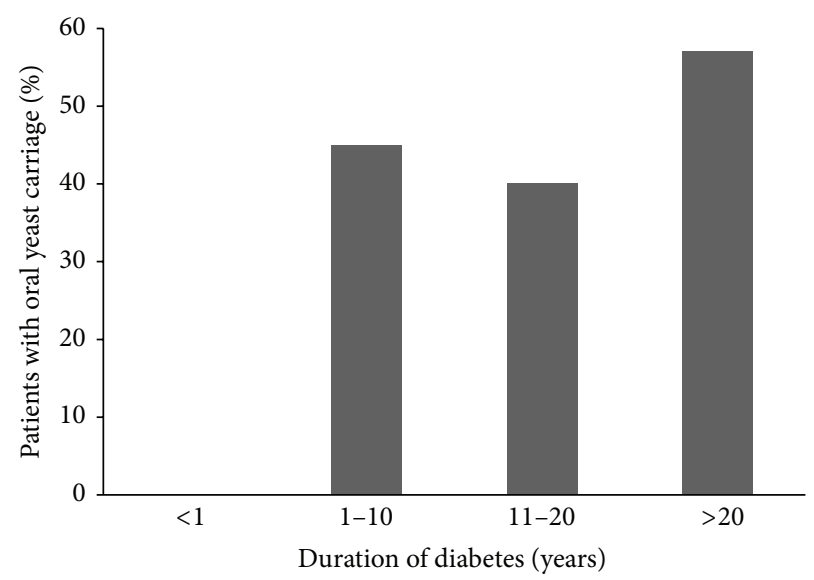

FIgURE 3: Oral yeast carriage in relation to duration of diabetes. 
TABLE 1: Spectrum of yeast species isolated in the diabetic and control groups.

\begin{tabular}{|c|c|c|c|c|c|}
\hline Yeast species & $\begin{array}{c}\text { Group } 1 \\
\text { (only on oral } \\
\text { antidiabetics) }\end{array}$ & $\begin{array}{c}\text { Group } 2 \\
\text { (only on insulin) }\end{array}$ & $\begin{array}{c}\text { Group } 3 \\
\text { (on both antidiabetics } \\
\text { and insulin) }\end{array}$ & Control Group & Number of Candida species \\
\hline C. albicans & 15 & 07 & 04 & 18 & 44 \\
\hline C. tropicalis & 02 & 02 & 04 & 01 & 09 \\
\hline C. krusei & 02 & 02 & 03 & - & 07 \\
\hline C. parapsilosis & 02 & 01 & 01 & 01 & 05 \\
\hline C. glabrata & - & 01 & 01 & 03 & 05 \\
\hline C. rugosa & - & 01 & - & - & 01 \\
\hline C. kefyr & - & 01 & - & - & 01 \\
\hline C. magnoliae & - & - & 01 & - & 01 \\
\hline Trichosporon asahi & - & 01 & - & - & 01 \\
\hline Number of isolates in each Group & 21 & 16 & 14 & 23 & TOTAL $=74$ \\
\hline
\end{tabular}

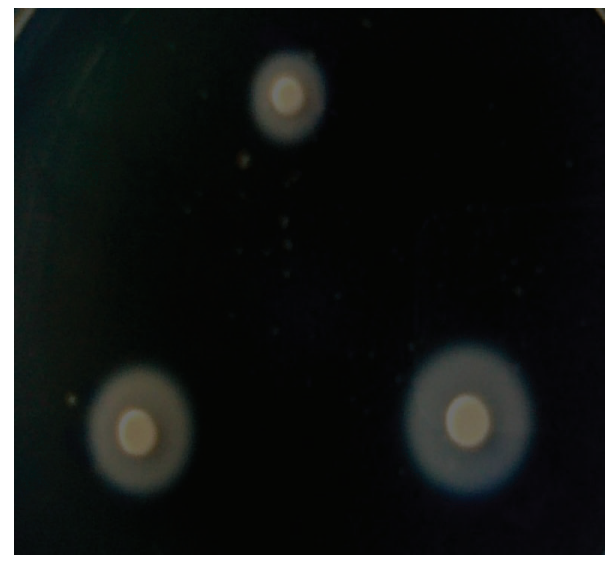

(a)

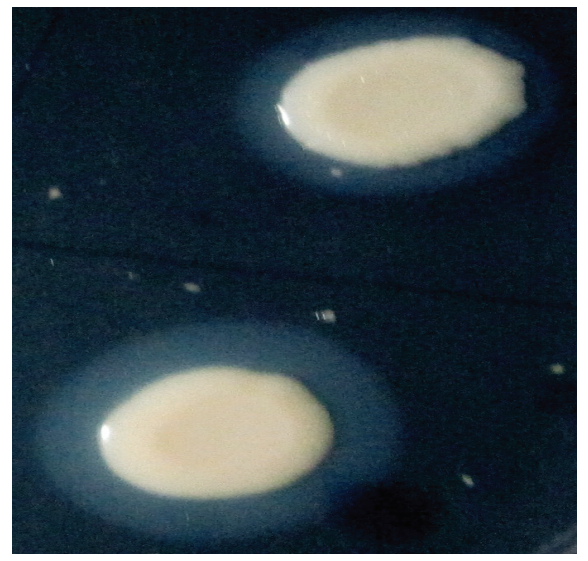

(b)

Figure 4: Production of proteinase (a) and phospholipase (b) by different Candida isolates.

similar findings. While, in DM group, fluconazole resistance was observed in $100 \%$ of C. krusei isolates, one isolate each of C. magnoliae, C. rugosa, and C. glabrata showed dose dependent susceptibility (Table 4 ).

In the present study $P_{z}$ value for proteinase ranged from 0.162 to 0.389 in diabetic group and 0.222 to 0.375 in control group $(P=0.034)$. Similar details for phospholipase producing isolates (only C. albicans) were found to be ranging from 0.346 to 0.455 in diabetic group and 0.400 to 0.478 in the control group $(P=0.018)$ (Table 5$)$.

Of the 26 C. albicans and 24 NCAC species, isolated from diabetic patients and tested for biofilm formation, positive findings were observed in 8 (31\%) C. albicans and 14 (58\%) NCAC species, as shown in Table 6. Among the isolates from control group, only $4(17.39 \%)$ were found to be positive for biofilm formation, which included 3 isolates of $C$. albicans and one $C$. parapsilosis $(P=0.027)$.

\section{Discussion}

Diabetes mellitus alters the cellular microenvironment in multiple organ systems including oral cavity. It is also known that oral candidiasis is a common opportunistic fungal infection in uncontrolled DM $[4,6]$. For complications like oropharyngeal candidiasis (OPC) in DM, source of infection is quantum of particular yeast residing as commensal in the oral cavity. In the present study though there was no clinical sign of OPC, $42 \%$ of diabetic patients had asymptomatic oral yeast carriage; this value is comparable to values reported in some of the earlier studies $[5,7,16]$. However, as expected, the carriage rate of yeast in the oral cavity of diabetic patients was found to be higher than that of control group corroborating with the earlier reports around the globe $[5,7,16]$, indicating that the oral microenvironment of DM patients supports the growth of opportunistic pathogens.

The present study yielded $47 \%$ NCAC species, which is higher than the values in earlier studies, which reported a range of $17-32 \%[7,16]$. A study conducted by Gonçalves et al. [17] on Brazilian population also reported a similar finding, with the carriage rate of NCAC as high as $39.8 \%$, compared to an earlier report in similar population. This difference in the percentage of isolation may be due to the difference in distribution of Candida in various geographical areas, the time of sampling, or the use of different methods 
TABLE 2: Oral carriage of Candida species in relation to fasting blood sugar.

\begin{tabular}{lccc}
\hline Fasting blood sugar level & Total number of patients & Number of patients yielded growth & Percentage (\%) \\
\hline$<110 \mathrm{mg} / \mathrm{dL}$ & 11 & 03 & 27.3 \\
$110-150 \mathrm{mg} / \mathrm{dL}$ & 42 & 17 & 40.5 \\
$151-200 \mathrm{mg} / \mathrm{dL}$ & 27 & 12 & 44.4 \\
$>200 \mathrm{mg} / \mathrm{dL}$ & 20 & 10 & 50.0 \\
\hline
\end{tabular}

TABLE 3: Oral carriage of Candida species in relation to age of diabetic patients.

\begin{tabular}{lcc}
\hline $\begin{array}{l}\text { Age group } \\
\text { (years) }\end{array}$ & $\begin{array}{c}\text { Total number } \\
\text { of patients }\end{array}$ & $\begin{array}{c}\text { Number of } \\
\text { patients yielded } \\
\text { growth }(\%)\end{array}$ \\
\hline $30-40$ & 17 & $07(41.17)$ \\
$41-50$ & 44 & $24(54.55)$ \\
$51-60$ & 27 & $09(33.33)$ \\
$61-70$ & 11 & $02(18.18)$ \\
$>70$ & 01 & 00 \\
\hline
\end{tabular}

for yeast recovery [18]. Interestingly, even now C. albicans is the predominant species isolated, accounting for $51 \%$ in diabetic patients, in concordance with earlier studies [5, $7,16]$. Another interesting point to note in the present study is the isolation of some rare species like C. magnoliae and Trichosporon asahii. Besides, by conventional methods, identity of these isolates was also confirmed by Vitek 2 YST system (bioMerieux, France).

The NCAC species are a heterogeneous group of yeasts that differ from each other and from C. albicans. Earlier, it was considered that $C$. albicans was the only species, causing infection [19]. However in recent years a shift towards NCAC has been reported, especially in haematological, transplanted, and intensive care unit (ICU) patients, often showing resistance to commonly used antifungal agents [20]. Also, study conducted at a major medical and research centre in New Delhi, India (including one of the present author, UB), on vulvovaginal candidiasis (VVC) in diabetic patients revealed an interesting scenario, where C. glabrata emerged as the primary NCAC species responsible for VVC in this group of patients [21-23]. Other NCAC species such as C. parapsilosis, C. krusei, C. dubliniensis, C. guilliermondii, C. lusitaniae, and C. rugosa, also have been reported as important emerging pathogens causing various clinical outcomes mainly in immunosuppressed patients [19].

While there are studies to show that candidal carriage is more in the oral cavity of diabetic patients, not many have investigated its relation to the duration of diabetes. The present study made an effort in this regard to understand the carriage rate of different yeast species in relation to the duration of diabetes. Interestingly, our study revealed that the presence of yeast in the oral cavity was directly related to the duration of DM. This may be due to the fact that prolonged diabetes leads to the impaired function of salivary glands causing a reduction of salivary flow and changes in salivary composition. This in turn leads to the rapid growth of Candida in these groups of patients [24]. But, more studies with larger number of patients are required to strengthen this fact further.

The present study also revealed a direct relation between oral candidal carriage and fasting blood sugar level, among diabetic patients (correlation coefficient, 0.970). Similar observation was reported by Khazal et al. [25] and LotfiKamran et al. [26]. The plausible explanation for this is the higher concentration of glucose in the blood and saliva, which in turn may enhance the adherence of yeast to buccal epithelial surface [27]. Also, in concurrence with previous reports, the present study could not establish a direct correlation between oral yeast carriage and the therapeutic modality or age of diabetic patients $[5,7]$.

Though emergence of drug resistance in C. albicans was reported [28], most of the studies revealed either nil or very low percentage of resistance in $C$. albicans isolated from different clinical specimens $[29,30]$. Hence, though none of the subjects in the study groups (patients with DM as also control group) in our study developed any clinical lesion in the oral cavity, despite carrying opportunistic pathogens, an in vitro antifungal susceptibility test of the isolates was still performed to obtain data on the susceptibility patterns and to determine an emerging trend, if any. The susceptibility testing revealed that the C. albicans and NCAC isolated from control group did not show resistance to any of the antifungal agents tested. C. albicans isolated from the diabetic group also yielded a similar finding (Table 2). Absence of drug resistance in these isolates can be attributed to the lack of several factors including degree of immunosuppression and prior exposure to a particular drug, acquiring of resistance gene, changes in the membrane lipid fluidity and asymmetry, the contribution of other chemotherapeutic drugs, and the intrinsic resistance of Candida species [31].

As is common among the NCAC species, resistance to Fluconazole was observed in all C. krusei isolates. One isolate each of C. magnoliae, C. rugosa, and C. glabrata showed dose dependent susceptibility. These resistant isolates can be described as primarily or intrinsically resistant strains [32-34]. It is to be noted here that none of the isolates showed resistance to other antifungal agents tested. However, this is only a one-time testing; the study should ideally be repeated with isolates from the same patients at different time intervals to observe any changes in the pattern of carriage and sensitivity to different antifungal agents [30], but here, as the patients were from outpatient department and did not have any clinical symptoms, they were lost to follow up.

The proteinase activity of Candida isolates from patients with DM and healthy controls often varies [35, 36]. The result in the present study was found to be in agreement with that of 
TABLE 4: Antifungal susceptibility pattern of the isolates.

\begin{tabular}{|c|c|c|c|c|}
\hline Species & Amphotericin B & Ketoconazole & Fluconazole & Voriconazole \\
\hline \multicolumn{5}{|c|}{ C. albicans $(n=44)$} \\
\hline \multirow{2}{*}{ Diabetes: 26} & S-26 & S-26 & S-26 & $S-26$ \\
\hline & $\mathrm{R}-0$ & $\mathrm{R}-0$ & $\mathrm{R}-0$ & $\mathrm{R}-0$ \\
\hline \multirow{2}{*}{ Healthy: 18} & S-18 & S-18 & S-18 & S-18 \\
\hline & $\mathrm{R}-0$ & $\mathrm{R}-0$ & $\mathrm{R}-0$ & $\mathrm{R}-0$ \\
\hline \multicolumn{5}{|c|}{ C. tropicalis $(n=9)$} \\
\hline \multirow{2}{*}{ Diabetes: 8} & S-8 & S-8 & S-8 & S-8 \\
\hline & $\mathrm{R}-0$ & $\mathrm{R}-0$ & $\mathrm{R}-0$ & $\mathrm{R}-0$ \\
\hline \multirow{2}{*}{ Healthy: 1} & S-1 & S-1 & S-1 & S-1 \\
\hline & $\mathrm{R}-0$ & $\mathrm{R}-0$ & $\mathrm{R}-0$ & $\mathrm{R}-0$ \\
\hline \multicolumn{5}{|l|}{ C. krusei $(n=7)$} \\
\hline \multirow{2}{*}{ Diabetes: 7} & S-7 & S-7 & S-0 & S-7 \\
\hline & $\mathrm{R}-0$ & $\mathrm{R}-0$ & $\mathrm{R}-7$ & $\mathrm{R}-0$ \\
\hline Healthy: 0 & - & - & - & - \\
\hline \multicolumn{5}{|c|}{ C. parapsilosis $(n=5)$} \\
\hline \multirow{2}{*}{ Diabetes: 4} & S-4 & S-4 & S-4 & S-4 \\
\hline & $\mathrm{R}-0$ & $\mathrm{R}-0$ & $\mathrm{R}-0$ & $\mathrm{R}-0$ \\
\hline \multirow{2}{*}{ Healthy: 1} & S-1 & S-1 & S-1 & S-1 \\
\hline & $\mathrm{R}-0$ & $\mathrm{R}-0$ & $\mathrm{R}-0$ & $\mathrm{R}-0$ \\
\hline \multicolumn{5}{|c|}{ C. glabrata $(n=5)$} \\
\hline \multirow{3}{*}{ Diabetes: 2} & S-2 & S-2 & S-1 & S-2 \\
\hline & & & SDD-1 & \\
\hline & $\mathrm{R}-0$ & $\mathrm{R}-0$ & $\mathrm{R}-0$ & $\mathrm{R}-0$ \\
\hline \multirow{2}{*}{ Healthy: 3} & S-3 & S-3 & S-3 & S-3 \\
\hline & $\mathrm{R}-0$ & $\mathrm{R}-0$ & $\mathrm{R}-0$ & $\mathrm{R}-0$ \\
\hline \multicolumn{5}{|c|}{ C. magnoliae $(n=1)$} \\
\hline \multirow{2}{*}{ Diabetes: 1} & S-1 & S-1 & SDD-1 & S-1 \\
\hline & $\mathrm{R}-0$ & $\mathrm{R}-0$ & $\mathrm{R}-0$ & $\mathrm{R}-0$ \\
\hline Healthy: 0 & - & - & - & - \\
\hline \multicolumn{5}{|l|}{ C. $\operatorname{kefyr}(n=1)$} \\
\hline \multirow{2}{*}{ Diabetes: 1} & S-1 & S-1 & S-1 & S-1 \\
\hline & $\mathrm{R}-0$ & $\mathrm{R}-0$ & $\mathrm{R}-0$ & $\mathrm{R}-0$ \\
\hline Healthy: 0 & - & - & - & - \\
\hline \multicolumn{5}{|l|}{ C. $\operatorname{rugosa}(n=1)$} \\
\hline \multirow{2}{*}{ Diabetes: 1} & S-1 & S-1 & SDD-1 & S-1 \\
\hline & $\mathrm{R}-0$ & $\mathrm{R}-0$ & $\mathrm{R}-0$ & $\mathrm{R}-0$ \\
\hline Healthy: 0 & - & - & - & - \\
\hline \multicolumn{5}{|l|}{ Others } \\
\hline \multicolumn{5}{|c|}{ Trichosporon asahii $(n=1)$} \\
\hline \multirow{2}{*}{ Diabetes: 1} & S-1 & S-1 & SDD-1 & S-1 \\
\hline & $\mathrm{R}-0$ & $\mathrm{R}-0$ & $\mathrm{R}-0$ & $\mathrm{R}-0$ \\
\hline Healthy: 0 & - & - & - & - \\
\hline
\end{tabular}

TABle 5: Proteinase and phosopholipase activity of Candida species isolated from healthy controls and diabetic group.

\begin{tabular}{lccc}
\hline Hydrolytic enzyme activity & Healthy controls & Diabetic group & $P$ value \\
\hline Proteinase $\left(\mathrm{P}_{\mathrm{z}}\right)$ & $0.302 \pm 0.040$ & $0.271 \pm 0.063$ & 0.034 \\
Phospholipase $\left(\mathrm{P}_{\mathrm{z}}\right)$ & $0.436 \pm 0.025$ & $0.409 \pm 0.033$ & 0.018 \\
\hline
\end{tabular}

Values expressed as mean \pm standard deviation (SD). 
TABLE 6: Biofilm positive Candida isolates from diabetic patients.

\begin{tabular}{|c|c|c|c|c|c|}
\hline \multirow{2}{*}{$\begin{array}{l}\text { Species } \\
\text { (No. of isolates) }\end{array}$} & \multirow{2}{*}{$\begin{array}{l}\text { Biofilm positive } \\
\text { (22) }\end{array}$} & \multicolumn{4}{|c|}{ Grade } \\
\hline & & $4+$ & $3+$ & $2+$ & $1+$ \\
\hline C. albicans (26) & 8 & 2 & 0 & 1 & 5 \\
\hline C. tropicalis (8) & 4 & 0 & 0 & 2 & 2 \\
\hline C. krusei (7) & 4 & 0 & 2 & 1 & 1 \\
\hline C. parapsilosis (4) & 4 & 0 & 2 & 2 & 0 \\
\hline C. glabrata (2) & 2 & 1 & 1 & 0 & 0 \\
\hline
\end{tabular}

Manfredi et al. [35], as the authors found that the proteinase activity was significantly higher in Candida species isolated from DM group than the control group ( $P$ value: $<0.05)$. All C. albicans isolated from both DM and control group were positive for phospholipase production. Earlier report on similar aspect did not show any significant difference in the phospholipase production among the isolates from DM and control group [36]. However, we observed an increased secretion of phospholipase enzyme in the isolates from the DM group ( $P$ value: $<0.05)$. This difference in observation may be due to strain to strain variation [37].

Reports on biofilm formation by Candida are available in literature [38] and it is known that there is a direct relationship between the ability to produce biofilm and its pathogenicity [39]. However, studies on biofilm formation on Candida isolates from type 2 diabetic patients are scanty. The present study showed that $58.33 \%$ NCAC species were capable of producing biofilm, whereas only $30 \%$ of C. albicans was capable of the same. Study conducted by Meurman et al. [19] on ICU patients also reported similar finding. Biofilm formation was found to be higher in isolates from diabetic group than the control group $(P=0.027)$.

Increased expression of these virulence factors in Candida isolated from diabetic group may be attributed to the process strain selection. This phenomenon has been reported as a reason for increased proteinase secretion in isolates from HIV seropositive individuals [40]. Due to strain selection original commensal strains get replaced by Candida populations that have more ability to cause disease. These strains can cause overt infection depending on the degree of immunosuppression of the patient.

Although it is a known fact that DM imposes an increased risk of oral candidiasis and that all C. albicans isolated from both the study groups in present study produced both proteinase and phospholipase enzymes, which is considered to be an important factor for the development of candidiasis, none of the subjects had clinical symptoms of oral candidiasis. The mechanism that limited proliferation in these cases cannot be explained by any single factor. One reason for this may be due to the fact that these patients were on appropriate therapeutic regimen and were on regular follow-up, which might have protected them from overt infection. Many other causes for the nondevelopment of overt infection by Candida species include blood group secretor status, salivary flow rates, antimicrobial constituents of saliva, lysozyme and lactoferrin release, $\beta$-defensins, presence of normal bacterial flora, and local immune system
[41]. Secretory immunoglobulin A (Ig A) and free secretory component (SC) in saliva also help in inhibiting the epithelial cell adhesion by Candida species [8]. Mucosal epithelium is considered as the first line of defence against invading pathogens [42]. Advanced research in immunology brought forth the active role played by epithelial cells in triggering immune response. It was reported that, upon recognition of the invading Candida species, epithelial cells secrete various antimicrobial peptides for the clearance or control of fungal infection directly. In addition to this, other immunological factors such as phagocytic cells, polymorphonuclear neutrophils, macrophages and dendritic cells, and several blood soluble factors like complement and antibody, also play an important role in the contribution of mucosal immunity to Candida infections [42].

To conclude, though $C$. albicans was the predominant species in the oral cavities of diabetic patients, the NCAC species seemed to be gaining significance, as some of these subjects also harboured fluconazole resistant species. Majority of these NCAC species were capable of producing biofilm. Also, secretion of hydrolytic enzymes and formation of biofilm were higher in Candida isolates from DM group than the control group. This is an alarming observation due to the fact that colonisation is an important fore-runner to the development of oral candidiasis eventually. The study highlights the importance of periodic monitoring of the spectrum of Candida species, especially NCAC species, in diabetic patients, their resistance patterns to frequently used antifungal agents, and their virulence attributes, as this will be of immense value in reducing the complications in this high-risk patient populations and also for the institution of appropriate control measures and treatment strategies.

\section{Conflict of Interests}

The authors declare that there is no conflict of interests regarding the publication of this paper.

\section{Acknowledgments}

The research group acknowledges, with thanks, the support rendered by Dr. Malini Capoor, Department of Microbiology, VMMC \& Safdarjung Hospital, New Delhi, Dr. Purva Mathur, Department of Laboratory Medicine, JPN Apex Trauma Centre, AIIMS, New Delhi, and Dr. Ravinder Kaur, Department of Microbiology, Maulana Azad Medical College, New Delhi. 


\section{References}

[1] S. R. Joshi and R. M. Parikh, "India-diabetes capital of the world: now heading towards hypertension," Journal of Association of Physicians of India, vol. 55, pp. 323-324, 2007.

[2] World Health Organization, Noncommunicable Diseases in the South-East Asia Region: Situation and Response, 2011.

[3] S. Wild, G. Roglic, A. Green, R. Sicree, and H. King, "Global prevalence of diabetes: estimates for the year 2000 and projections for 2030," Diabetes Care, vol. 27, no. 5, pp. 1047-1053, 2004.

[4] A. Y. Al-Maskari, M. Y. Al-Maskari, and S. Al-Sudairy, "Oral manifestations and complications of diabetes mellitus: a review," Sultan Qaboos University Medical Journal, vol. 11, no. 2, pp. 179186, 2011.

[5] B. V. Kumar, N. S. Padshetty, K. Y. Bai, and M. S. Rao, "Prevalence of Candida in the oral cavity of diabetic subjects," Journal of Association of Physicians of India, vol. 53, pp. 599-602, 2005.

[6] L. Shirmali, M. Astekar, and G. V. Sowmya, "Correlation of oral manifestations in controlled and uncontrolled diabetes mellitus," International Journal of Oral and Maxillofacial Pathology, vol. 2, no. 4, pp. 24-27, 2011.

[7] S. A. Al-Attas and S. O. Amro, "Candidal colonization, strain diversity, and antifungal susceptibility among adult diabetic patients," Annals of Saudi medicine, vol. 30, supplement 2, pp. 101-108, 2010.

[8] N. S. Soysa, L. P. Samaranayake, and A. N. B. Ellepola, "Diabetes mellitus as a contributory factor in oral candidosis," Diabetic Medicine, vol. 23, no. 5, pp. 455-459, 2006.

[9] L. J. R. Milne, "Fungi," in Mackie and McCartney. Practical Medical Microbiology, J. G. Collee, J. P. Duguid, A. G. Fraser, and B. P. Marmion, Eds., pp. 675-699, Churchill Livingstone, 13th edition, 1989.

[10] M. Slifkin, "Tween 80 opacity test responses of various Candida species," Journal of Clinical Microbiology, vol. 38, no. 12, pp. 4626-4628, 2000.

[11] M. J. Maxwell, S. A. Messer, R. J. Hollis et al., "Evaluation of Etest method for determining fluconazole and voriconazole MICs for 279 clinical isolates of Candida species infrequently isolated from blood," Journal of Clinical Microbiology, vol. 41, no. 3, pp. 1087-1090, 2003.

[12] Clinical and Laboratory Standards Institute, "Reference method for broth dilution antifungal susceptibility testing of yeasts, approved Standard-third edition,", CLSI Document M27-A3, Clinical and Laboratory Standards Institute (CLSI), 2008.

[13] S. Yousuf, A. Ahmad, A. Khan, N. Manzoor, and L. A. Khan, "Effect of garlic-derived allyl sulphides on morphogenesis and hydrolytic enzyme secretion in Candida albicans," Medical Mycology, vol. 49, no. 4, pp. 444-448, 2011.

[14] S. Shreaz, R. Bhatia, N. Khan et al., "Cinnamic aldehydes affect hydrolytic enzyme secretion and morphogenesis in oral Candida isolates," Microbial Pathogenesis, vol. 52, no. 5, pp. 251258, 2012.

[15] M. Tumbarello, B. Posteraro, E. M. Trecarichi et al., "Biofilm production by Candida species and inadequate antifungal therapy as predictors of mortality for patients with candidemia," Journal of Clinical Microbiology, vol. 45, no. 6, pp. 1843-1850, 2007.

[16] M. Bharathi, A. U. Rani, and C. Sandhya, "A comparative study of carrier state of Candida and its speciation in oral flora among healthy individuals, persons with DM and HIV seropositive individuals," Our Dermatology online, vol. 3, supplement 2, pp. 102-106, 2012.
[17] R. H. P. Gonçalves, E. T. Miranda, J. E. Zaia, and M. J. S. M. Giannini, "Species diversity of yeast in oral colonization of insulin-treated diabetes mellitus patients," Mycopathologia, vol. 162, no. 2, pp. 83-89, 2006.

[18] J. Xu and T. G. Mitchell, "Geographical differences in human oral yeast flora," Clinical Infectious Diseases, vol. 36, no. 2, pp. 221-224, 2003.

[19] J. H. Meurman, E. Siikala, M. Richardson, and R. Rautemaa, "Non-Candida albicans Candida yeast of the oral cavity," in Communicating Current Research and Educational Topics and Trends in Applied Microbiology, A. Mendez-Vilas, Ed., pp. 719731, Formatex, 2007.

[20] R. Kaur, R. Goyal, M. S. Dhakad, P. Bhalla, and R. Kumar, "Epidemiology and virulence determinants including biofilm profile of Candida infections in an ICU in a tertiary hospital in India," Journal of Mycology, vol. 2014, Article ID 303491, 8 pages, 2014.

[21] R. Goswami, V. Dadhwal, S. Tejaswi et al., "Species-specific prevalence of vaginal candidiasis among patients with diabetes mellitus and its relation to their glycaemic status," Journal of Infection, vol. 41, no. 2, pp. 162-166, 2000.

[22] D. Goswami, R. Goswami, U. Banerjee et al., "Pattern of Candida species isolated from patients with diabetes mellitus and vulvovaginal candidiasis and their response to single dose oral fluconazole therapy," Journal of Infection, vol. 52, no. 2, pp. 111-117, 2006.

[23] D. Ray, R. Goswami, U. Banerjee et al., "Prevalence of Candida glabrata and its response to boric acid vaginal suppositories in comparison with oral fluconazole in patients with diabetes and vulvovaginal candidiasis," Diabetes Care, vol. 30, no. 2, pp. 312 317, 2007.

[24] C. Negrato and O. Tarzia, "Buccal alterations in diabetes mellitus," Diabetology \& Metabolic Syndrome, vol. 2, no. 1, article 3, 2010.

[25] F. A. Khazal, A. Mahan, and H. H. Al-Hasnawi, "Oral carriage rate of Candida species in diabetic patients," Al-Kindy College Medical Journal, vol. 3, no. 1, pp. 8-11, 2006.

[26] M. H. Lotfi-Kamran, A. A. Jafari, A. Falah-Tafti, A. Tavakoli, and M. H. Falah Zadeh, "Candida colonisation on the denture of diabetic and non-diabetic patients," Dental Research Journal, vol. 6, no. 1, pp. 23-27, 2009.

[27] A. M. L. Pedersen, "Diabetes mellitus and related oral manifestations," Oral Biosciences and Medicine, vol. 4, no. 1, pp. 229-248, 2004.

[28] M. Ruhnke, A. Eigler, I. Tennagen, B. Geiseler, E. Engelmann, and M. Trautmann, "Emergence of fluconazole-resistant strains of Candida albicans in patients with recurrent oropharyngeal candidosis and human immunodeficiency virus infection," Journal of Clinical Microbiology, vol. 32, no. 9, pp. 2092-2098, 1994.

[29] V. S. Rathod, J. S. Raut, and S. Mohan Karuppayil, "In vitro antifungal susceptibility reveals occurrence of azole resistance among clinical isolates of Candida albicans," Asian Journal of Pharmaceutical and Clinical Research, vol. 5, no. 3, pp. 170-173, 2012.

[30] A. A. Lattif, U. Banerjee, R. Prasad et al., "Susceptibility pattern and molecular type of species-specific Candida in oropharyngeal lesions of Indian Human immunodeficiency Virus-Positive patients," Journal of Clinical Microbiology, vol. 42, no. 3, pp. 1260-1262, 2004.

[31] A. A. Lattif, "Molecular typing and in vitro fluconazole susceptibility of Candida species isolated from diabetic and nondiabetic 
women with vulvovaginal candidiasis in India," Journal of Microbiology, Immunology and Infection, vol. 44, no. 3, pp. 166171, 2011.

[32] M. A. Pfaller, D. J. Diekema, D. L. Gibbs et al., "Candida krusei, a multidrug-resistant opportunistic fungal pathogen: geographic and temporal trends from the ARTEMIS DISK Antifungal Surveillance Program, 2001 to 2005," Journal of Clinical Microbiology, vol. 46, no. 2, pp. 515-521, 2008.

[33] M. A. Pfaller, D. J. Diekema, A. L. Colombo et al., "Candida rugosa, an emerging fungal pathogen with resistance to azoles: geographic and temporal trends from the ARTEMIS DISK Antifungal Surveillance Program," Journal of Clinical Microbiology, vol. 44, no. 10, pp. 3578-3582, 2006.

[34] J.-P. Vermitsky and T. D. Edlind, "Azole resistance in Candida glabrata: coordinate upregulation of multidrug transporters and evidence for a Pdrl-like transcription factor," Antimicrobial Agents and Chemotherapy, vol. 48, no. 10, pp. 3773-3781, 2004.

[35] M. Manfredi, M. J. McCullough, Z. M. Al-Karaawi, P. Vescovi, and S. R. Porter, "In vitro evaluation of virulence attributes of Candida spp. isolated from patients affected by diabetes mellitus," Oral Microbiology and Immunology, vol. 21, no. 3, pp. 183-189, 2006.

[36] C. S. P. Tsang, F. C. S. Chu, W. K. Leung, L. J. Jin, L. P. Samaranayake, and S. C. Siu, "Phospholipase, proteinase and haemolytic activities of Candida albicans isolated from oral cavities of patients with type 2 diabetes mellitus," Journal of Medical Microbiology, vol. 56, no. 10, pp. 1393-1398, 2007.

[37] M. A. Ghannoum, "Potential role of phospholipases in virulence and fungal pathogenesis," Clinical Microbiology Reviews, vol. 13, no. 1, pp. 122-143, 2000.

[38] W. J. da Silva, J. Seneviratne, N. Parahitiyawa, E. A. R. Rosa, L. P. Samaranayake, and A. A. del Bel Cury, "Improvement of XTT assay performance for studies involving Candida albicans biofilms," Brazilian Dental Journal, vol. 19, no. 4, pp. 364-369, 2008.

[39] L. Sherry, R. Rajendran, D. F. Lappin et al., "Biofilms formed by Candida albicans bloodstream isolates display phenotypic and transcriptional heterogeneity that are associated with resistance and pathogenicity," BMC Microbiology, vol. 14, article 182, 2014.

[40] M. W. Ollert, C. Wende, M. Gorlich et al., "Increased expression of Candida albicans secretory proteinase, a putative virulence factor, in isolates from human immunodeficiency virus-positive patients," Journal of Clinical Microbiology, vol. 33, no. 10, pp. 2543-2549, 1995.

[41] C. R. Costa, A. J. Cohen, O. F. L. Fernandes et al., "Asymptomatic oral carriage of Candida species in HIV-infected patients in the highly active antiretroviral therapy era," Revista do Instituto de Medicina Tropical de Sao Paulo, vol. 48, no. 5, pp. 257-261, 2006.

[42] S. C. Cheng, L. A. B. Joosten, B. J. Kullberg, and M. G. Netea, "Interplay between Candida albicans and the mammalian innate host defense," Infection and Immunity, vol. 80, no. 4, pp. 1304-1313, 2012. 

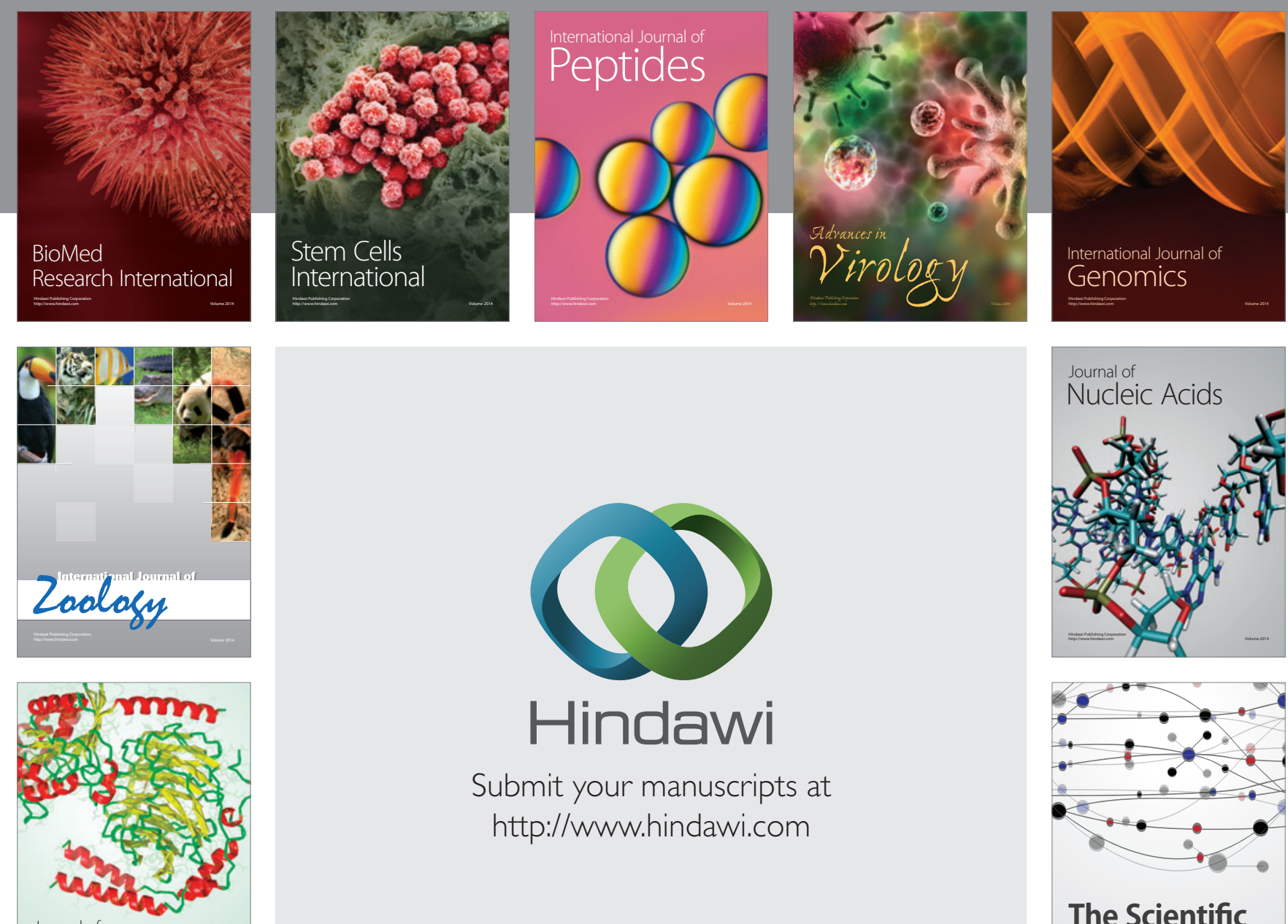

Submit your manuscripts at

http://www.hindawi.com

Journal of
Signal Transduction
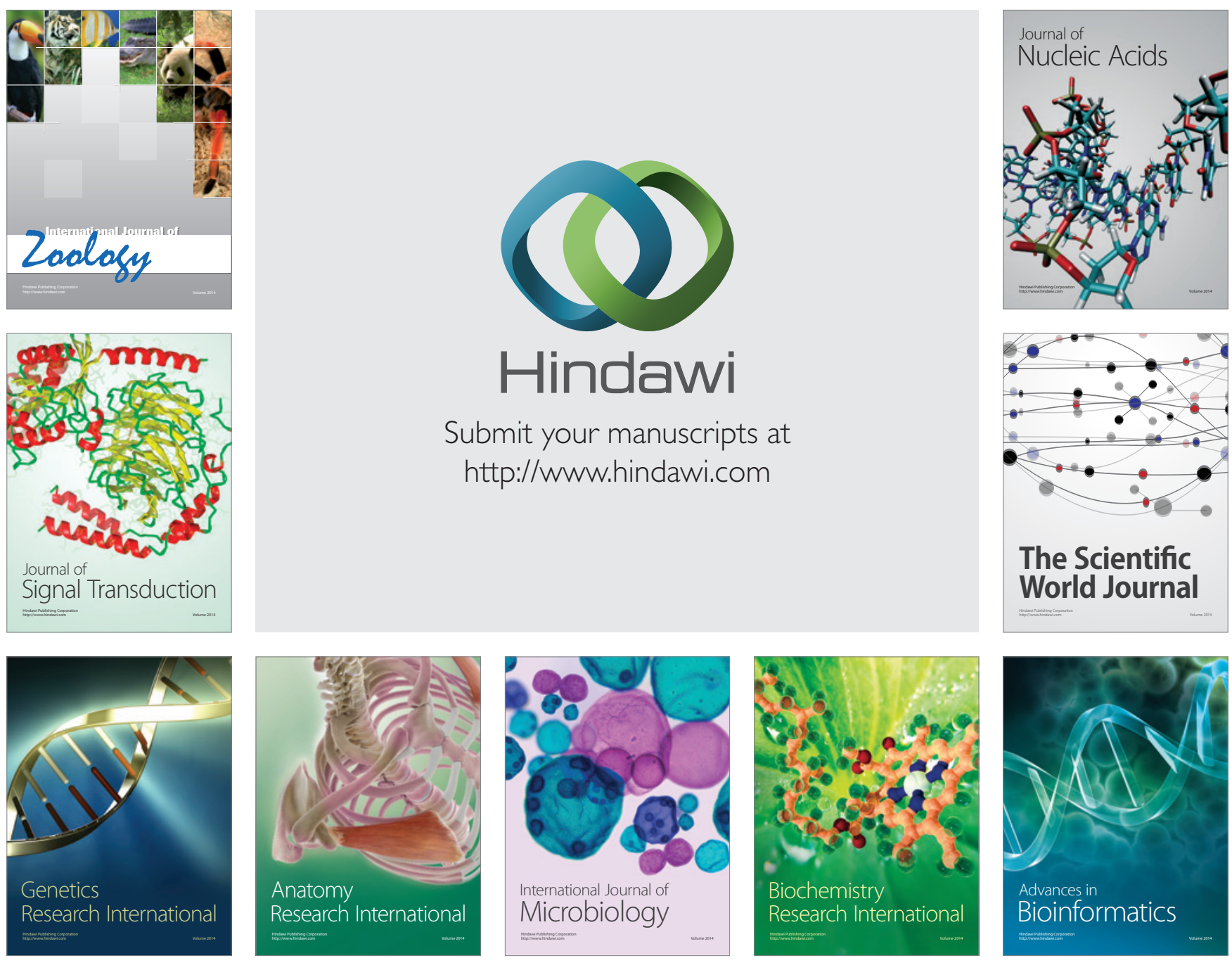

The Scientific World Journal
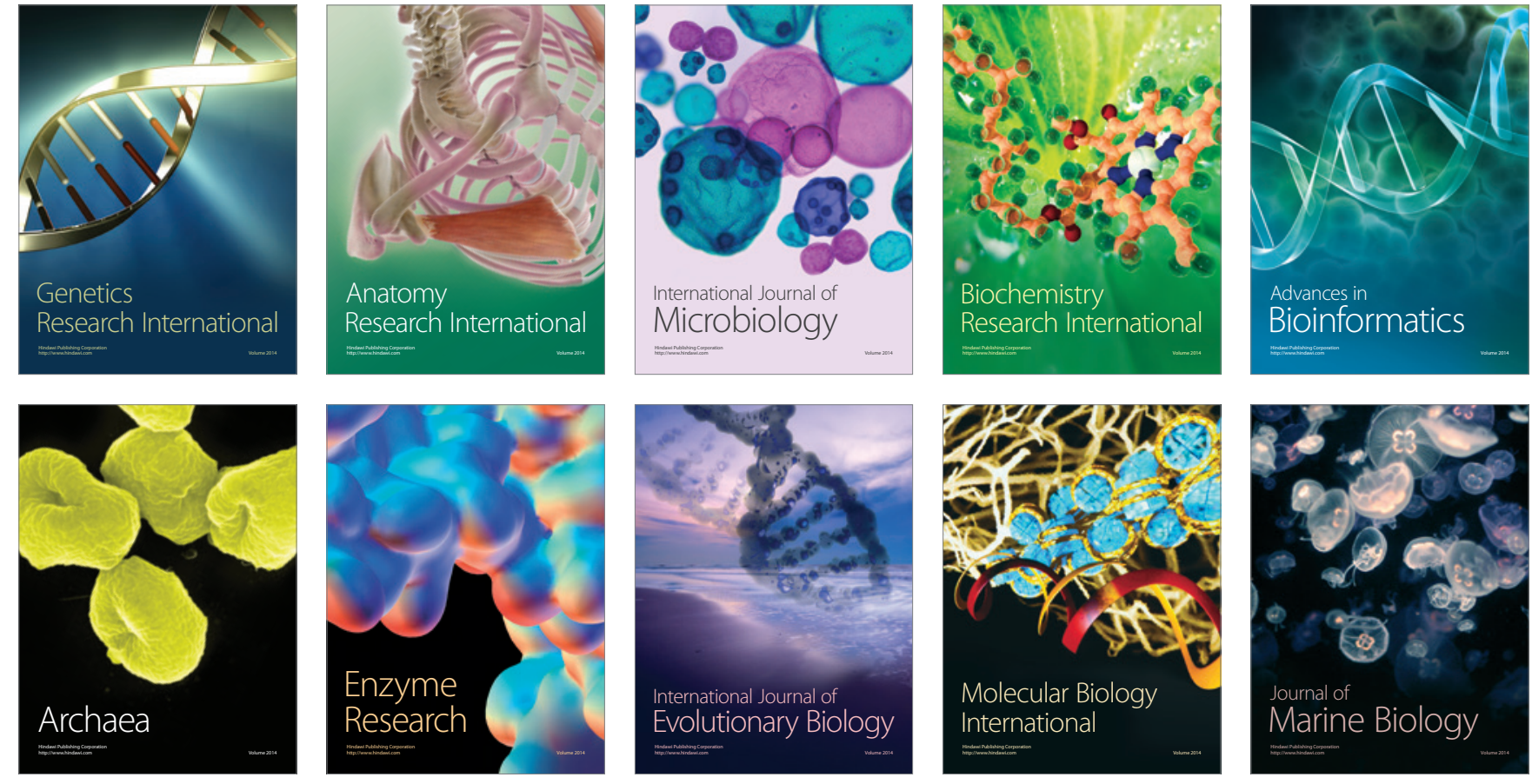